\title{
Desempleo en América Latina:
}

\section{¿FLEXIBILIDAD LABORAL O ACUMULACIÓN DE CAPITAL?}

\author{
Luis Fernando García y Moritz Cruz
}

Fecha de recepción: 19 de octubre de 2016. Fecha de aceptación: 13 de enero de 2017.

\begin{abstract}
RESUMEN
El desempleo en América Latina ha tenido una disminución sustancial durante el periodo 2000-2013. Desde el punto de vista teórico convencional, lo anterior sería consecuencia de las reformas orientadas a flexibilizar el mercado de trabajo. Por otro lado, la visión post-keynesiana sustentaría que la caída de la tasa de desempleo se debe al aumento de la acumulación de capital. El presente documento intenta conocer cuál de estas visiones teóricas es apoyada por la evidencia empírica. Los resultados del panel de datos sugieren que la acumulación de capital es la variable que explica mayormente el desempeño del desempleo. La recomendación de política de esta evidencia es mantener en crecimiento la inversión a través de instrumentos que estimulen la demanda efectiva. Palabras clave: desempleo, mercado de trabajo, datos de panel, teoría post-keynesiana, teoría neoclásica.
\end{abstract}

Clasificación JEL: E12, E13, E24, J64, N36.

\section{Unemployment in Latin America: Labor Market Flexibility or Capital ACCuMulation?}

\begin{abstract}
Unemployment in Latin America fell substantially in 2000-2013, which, from the standpoint of conventional theory, would be the outcome of reforms designed to make the labor market more flexible. On the other hand, a post-keynesian perspective would maintain that a dropping unemployment rate is due to rising capital accumulation. This paper aims to determine which of these theoretical visions is supported by the empirical evidence. The panel data results suggest that capital accumulation is the variable that explains the majority of the behavior of unemployment. The policy recommendation based on this evidence is to keep investment growing via instruments that stimulate effective demand.
\end{abstract}

Key Words: Unemployment, labor market, panel data, post-keynesian theory, neoclassical theory.

${ }^{1}$ Instituto de Investigaciones Económicas, Universidad Nacional Autónoma de México (UNAM), México. Correos electrónicos: luis_gacov@hotmail.com y aleph398@gmail.com, respectivamente. 


\section{CHÔMAGE EN AMÉRIQUE LATINE: FLEXIBILITÉ DU TRAVAIL OU ACCUMULA- TION DU CAPITAL? \\ Résumé}

Le chômage en Amérique Latine a subit une diminution substantielle durant la période 2000-2013. Du point de vue théorique conventionnel, ce qui précède serait le résultat des reformes orientés vers l'assouplissement du marché du travail. En revanche, le point de vue post-keynésien soutiendrait l'idée que la baisse du taux de chômage est due à une augmentation de l'accumulation du capital. Le présent document cherche à savoir lequel de ces points de vue théoriques est soutenue par des preuves empiriques. Les résultats du panneau de données suggèrent que l'accumulation du capital est la variable qui explique largement la performance du chômage. La recommandation de politique de cette preuve est de garder le même rythme de croissance de l'investissement à travers des instruments qui stimulent la demande effective.

Mots clés: chômage, marché du travail, panneau de données, théorie post-keynésienne, théorie néoclassique.

\section{DESEMPREGO NA AMÉRICA LATINA: ¿̨FLEXIBILIDADE TRABALHISTA OU ACU- MULAÇÁO DE CAPITAL? \\ Resumo}

O desemprego na América Latina teve uma diminuição substancial durante o período 2000-2013. Desde o ponto de vista teórico convencional, o anterior seria consequência das reformas orientadas a flexibilizar do mercado de trabalho. Por outro lado, a visão pós-keynesiana sustentaria que a queda da taxa de desemprego se deve ao aumento da acumulação de capital. O presente documento tenta conhecer qual destas visóes teóricas é apoiada pela evidência empírica. Os resultados do panel de dados sugerem que a acumulação de capital é a variável que explica em maior medida o desempenho do desemprego. A recomendação de política desta evidência é manter em crescimento o investimento através de instrumentos que estimulem a demanda efetiva.

Palavras-chave: desemprego, mercado de trabalho, dados de painel, teoria pós-keynesiana, teoria neoclássica.

\section{拉丁美洲的失业情况：劳动灵活性还是资本积累?}

路易斯・费尔南多 - 加西亚莫利斯 - 科鲁兹

摘要

2000-2013年期间拉丁美洲的失业率显著下降。从传统的理论角度来看, 失业率下降是旨在调节劳动市场的一系列改革的结果。另一方面, 后凯恩 斯主义观点认为失业率的下降归功于资本积累的增加。本文旨在研究哪种 理论观点有经验证据的支撑。研究数据表明资本的积累是能够最大限度阐 释失业率下降的变量。因此我们推荐采取刺激实际需求措施以保证投资增 长。

关键词: 失业劳动市场 数据板后凯恩斯理论 新古典理论 


\section{INTRODUCCIÓN}

Durante el periodo 2000-2013, el desempleo en América Latina declinó lenta, pero sostenidamente, yendo de una tasa de $9.3 \%$ en 2003 a una de $6.2 \%$ en 2013. Este hecho destacable no había sido observado en el pasado reciente. De manera interesante, tal comportamiento estuvo marcado por dos hechos estilizados que potencialmente pueden explicarlo, al poder vincular cada uno de ellos con una visión teórica sobre el origen del desempleo.

Por un lado, desde la década de los noventa del siglo pasado, a lo largo de la región se implementaron reformas laborales cuyo fin consistió en flexibilizar el mercado de trabajo (Vega, 2005). Es decir, el pasado reciente estuvo marcado por la modificación de las diversas legislaciones laborales con la finalidad de que la oferta y la demanda laboral se ajustaran a su nivel de equilibrio, entendido como aquél consistente con la tasa natural, potencial o tasa de desempleo no-inflacionaria. Remover la rigidez del mercado laboral, en la visión teórica neoclásica o de los llamados nuevos keynesianos, es fundamental para reducir el nivel de desempleo. En este sentido, la disminución del desempleo observado podría ser el resultado de las reformas implementadas para flexibilizar el mercado laboral.

Por otra parte, tanto la demanda agregada como la acumulación de capital, entendida como el aumento de la inversión bruta fija total, mantuvieron durante el periodo señalado un dinamismo muy superior al de la década de los ochenta, pero similar al de la de los noventa, con una tasa promedio anual de 3.2 y de $4.8 \%$ en el caso de la demanda agregada y de la inversión, respectivamente; esto incluyendo 2009, año que capitalizó la contracción económica derivada de la Gran Recesión. Como porcentaje del producto interno bruto (PIB), no obstante, la acumulación de capital, sí registró un incremento significativo, aumentando cuatro puntos porcentuales entre 2002 y 2008, pasando de 17 a $21 \%$ del PIB. En la visión teórica post-keynesiana, la demanda efectiva, a través de su impacto en las decisiones de inversión de los empresarios, es la que determina los niveles de (des)empleo. Así, la elevada dinámica de la demanda y la acumulación de capital durante el periodo permitirían sugerir que fue ésta la que causó la disminución de la tasa de desempleo, dando lugar a una explicación post-keynesiana.

Ambos hechos estilizados permiten establecer la siguiente interrogante: ¿qué explica la paulatina disminución del desempleo en la región: la flexibilidad laboral o el dinamismo de la demanda y de la tasa de acumulación? Encontrar la respuesta adecuada, flexibilidad laboral o demanda efectiva, no es trivial, toda vez que se podría sugerir con mayor certeza la clase de política a 
enfatizar para mantener la tendencia a la baja del desempleo: sea profundizar la flexibilización del mercado laboral o bien orientar los esfuerzos a mantener al alza la demanda y, consecuentemente, la acumulación de capital. Asimismo, recordemos que el desempleo se cuenta entre las principales fuentes de pobreza, y en la medida en que el primero logre reducirse consistentemente, la batalla contra el segundo se facilitará.

El objetivo de este trabajo es entonces intentar dar respuesta a dicha interrogante para el caso latinoamericano, algo que hasta donde sabemos no ha sido hecho. $Y$ en este sentido, aunque el debate sobre el origen del empleo es relativamente añejo, su estudio para América Latina es actual. Para alcanzar el objetivo planteado se realizaron estimaciones del desempleo con datos panel para comprobar empíricamente cada uno de los argumentos teóricos señalados. Los datos provienen de un conjunto de 18 economías latinoamericanas para el periodo 2000-2013.

El trabajo está estructurado de la siguiente forma: la segunda sección describe brevemente el sustento teórico convencional y post-keynesiano acerca de la determinación del nivel de (des)empleo; la tercera sección presenta y discute los resultados empíricos del ejercicio econométrico, y la última sección presenta las conclusiones.

\section{El (des)empleo en las visiones teóricas neoclásica y post-keynesiana}

En esta sección se describe cómo las visiones teóricas neoclásica y post-keynesiana argumentan sobre la determinación del nivel de (des)empleo. La intención de esto es doble. En primera instancia poder asociar teóricamente la tendencia a la baja del desempleo en América Latina durante la década del 2000 con la explicación del (des)empleo de cada escuela; en segundo lugar, visualizar las diferencias entre ambos enfoques para comprender las recomendaciones de política que emanan de cada uno de ellos.

\section{El(des)empleo en la visión teórica neoclásica}

La perspectiva teórica convencional, neoclásica o de las llamados nuevos keynesianos (Hicks, 1937; Samuelson y Solow, 1960; Phelps, 1972; Blanchard, 1997, entre otros), propone que en el mercado laboral se determina el nivel de empleo como resultado de las decisiones de maximización independientes 
pero relacionadas de, por un lado, los empresarios sobre su ganancia y, por el otro, de los consumidores sobre su utilidad (en términos de capacidad de consumo). Así, los empresarios demandarán fuerza laboral hasta el punto en que el costo de ésta iguale su productividad marginal, mientras la fuerza laboral se ofertará hasta que la desutilidad marginal del ocio iguale el beneficio por trabajar. Asumiendo perfecta flexibilidad de dicho mercado, el salario se encargará de igualar la oferta y la demanda laboral, limpiando dicho mercado. Este nivel se asume de pleno empleo y está asociado con el producto potencial o natural, o con su contraparte: la llamada tasa de desempleo no aceleradora de la inflación.

Por lo anterior, en el caso de intentar aumentar la demanda agregada para disminuir el desempleo por debajo de dicha tasa el resultado será “....el crecimiento de los precios. La razón de esto es el creciente poder de los sindicatos para negociar mayores salarios conforme crece el empleo" (Blanchard, 1997: 279). Al producto o tasa natural, la economía converge de manera inexorable y aunque puede sufrir desviaciones de dicho punto, regresará al mismo por las mismas fuerzas que la llevaron ahí: la intención de máximo beneficio de los agentes y la flexibilidad del salario. Esto conlleva la idea de que, una vez que el desempleo alcanza su tasa natural, dada por factores estructurales y las características del mercado de trabajo, no tiene sentido intentar reducirlo a través de políticas alternativas a las de dicho mercado porque en caso de hacerlo el desempleo tenderá a regresar a dicha tasa, aunque con efectos colaterales adversos en la economía, como una espiral inflacionaria.

Dado que las fuerzas de mercado aseguran el nivel de pleno empleo, el modelo sugiere la nula posibilidad para el desempleo involuntario. Todo aquel desempleado lo está por razones friccionales, estructurales, cíclicas o por su propia voluntad. Todos estos trabajadores son los que integran la tasa de desempleo no inflacionaria.

Sin embargo, sostiene la teoría, cuando el mercado laboral enfrenta rigideces institucionales, el empleo se ubicará por debajo de la tasa de equilibrio natural, o dicho de otra manera, la tasa de desempleo estará por encima de tasa de desempleo no aceleradora de la inflación. Lo anterior implica que el costo del factor trabajo se ubique por encima de aquel consistente con su productividad, impidiendo que otros trabajadores puedan ser empleados. ${ }^{2} \mathrm{De}$ aquí se sigue que el desempleo que no es friccional, estructural o cíclico está

\footnotetext{
El mayor costo del factor trabajo también es visto como una pérdida de competitividad en los mercados internacionales en esta perspectiva. De ahí también que la flexibilidad laboral sea vista como un mecanismo para recuperar dicha competitividad.
} 
explicado principal (y casi exclusivamente) por la inflexibilidad del mercado laboral (Bernal-Verdugo, 2012, 2013; Layard et al., 1991). De esta manera, cuanto más rígida sea la estructura institucional del mercado laboral (reflejada, entre otras, en la existencia de beneficios al desempleo, de restricciones para despedir, de salarios mínimos, de sindicatos, de impuestos) mayor es la dificultad de los empresarios para incrementar la demanda por trabajo, aunque los trabajadores tengan incentivos para mantenerse en o incorporarse al mercado laboral formal. Ambos factores dan como resultado un mayor nivel de desempleo del que existiría de contar con condiciones de plena flexibilidad.

De esta línea de razonamiento se deriva que incluso choques exógenos que elevan la tasa natural de desempleo o no aceleradora de la inflación, originando así mayores (y persistentes o permanentes) niveles de desempleo, tienen también como sustento último la rigidez del mercado laboral. En otras palabras, el reconocido fenómeno de histéresis del desempleo tiene como explicación fundamental la inflexibilidad laboral. En este caso la lógica más argumentada se centra en el poder de un grupo de trabajadores denominados insiders, para negociar y establecer, además de otros beneficios, un salario real por encima del de mercado, impidiendo así que los desempleados, denominados outsiders, se incorporen (aunque lo deseen), al mercado laboral (Blanchard, 1997; Carlin y Soskice, 2006).

Desde esta perspectiva, la solución al problema del desempleo creciente y persistente consiste en implementar mecanismos para flexibilizar el mercado laboral, entiéndase como la eliminación de cualquiera de las barreras que impide a los empresarios aumentar su demanda laboral. Es decir, se deben remover o reducir la existencia de beneficios al desempleo, de restricciones para despedir, de salarios mínimos, de sindicatos, entre otras. Lo anterior implica generalmente reformar en dicha dirección la legislación laboral de las economías.

Es importante señalar que la flexibilización del mercado laboral no es nueva y ha sido abrazada tanto por países avanzados como en desarrollo. En este sentido, existe amplia literatura empírica sobre sus resultados, apuntando a una conclusión ambigua sobre la efectividad de la flexibilidad laboral para abatir el desempleo, toda vez que hay trabajos cuyos resultados la apoyan al tiempo que los de otros la refutan. Por ejemplo, gran parte de la literatura empírica enfocada a explicar el elevado desempleo en Europa a partir de los ańos setenta (Layard et al., 1991; Siebert, 1997, entre otros), no ha podido demostrar consistentemente que la inflexibilidad del mercado laboral sea su causa principal (Arestis et al., 2007: 126, y trabajos ahí citados). En particular, 
Nickell et al. (2005) señalan que el alto desempleo en Europa desde la década de los sesenta hasta mediados de los ańos noventa puede atribuirse en $50 \%$ a cambios institucionales en el mercado de trabajo y el otro $50 \%$ a insuficiencia de la demanda efectiva.

La literatura más reciente, estudiando diversos países tanto avanzados como en desarrollo, afirma que hay evidencia robusta sobre la existencia de una relación inversa entre flexibilidad de los mercados laborales y las tasas de desempleo. En adición, dicha literatura enfatiza que la mayor flexibilidad de los mercados laborales se relaciona positivamente con las elasticidades de empleo (Bernal-Verdugo et al., 2012, 2013, y las citas ahí contenidas; Crivelli et al., 2012; Furceri, 2012). Toda esta literatura, emanada del Fondo Monetario Internacional (FMI), ha sido cuestionada por Aleksynska (2014) en sus aspectos metodológicos sobre las series de tiempo usadas, señalando que si tales aspectos metodológicos son tomados en cuenta, no es posible replicar sus resultados, dejando su robustez en cuestionamiento.

Hay, por otro lado, trabajos que mantienen una postura más bien cautelosa sobre los beneficios y costos de algunas regulaciones laborales, como el salario mínimo o la legislación laboral, al beneficiar en algunos aspectos, como mejorar la distribución del ingreso, pero tener mínimo o nulo impacto en otros, como en la productividad (Betcherman, 2014). En el contexto de una unión monetaria, se ha encontrado que la flexibilidad laboral reduce el bienestar económico (Galí y Monacelli, 2016). En suma, como destacamos, de la evidencia empírica arrojada por la literatura no puede extraerse una conclusión uniforme sobre los beneficios de la flexibilización laboral en el (des) empleo.

Finalmente, la literatura sobre histéresis arroja también resultados ambiguos sobre la efectividad o no de la flexibilidad laboral para abatir el desempleo (Cross et al., 1990; Maurer y Nivia, 1994; Dias, 1995; León-Ledesma y McAdam, 2004; Cheng-Hsun et al., 2008; Mednik et al., 2012; Ayala et al., 2012; Dritsaki y Dritsaki, 2013). Lo anterior debido a que a lo largo de estos trabajos no siempre se confirma que la tasa de desempleo sea no estacionaria (a través del rechazo de las hipótesis de raíz unitaria de la serie en cuestión). Es decir, si acaso la tasa de desempleo fuera estacionaria, entonces ante un choque, la serie se revertiría a su media (digamos a la tasa de desempleo no aceleradora de la inflación), derivándose que las posibles rigideces del mercado laboral no son un factor que le impiden volver a su media. 


\section{El (des)empleo en la visión teórica post-keynesiana}

A la propuesta neoclásica y nueva keynesiana sobre la forma en que se determina el nivel de (des)empleo en una economía se contrapone la visión teórica post-keynesiana, uno de cuyos pilares sostiene que la flexibilidad del salario (nominal o real) ${ }^{3}$ no juega papel alguno para comprender los niveles de (des) empleo en las economías capitalistas (Keynes, 2000; Davidson, 2007). ${ }^{4}$ Más específicamente, se argumenta que la oferta laboral no es función del salario real. Eliminada así la explicación del desempleo por el lado del mercado laboral neoclásico, esta propuesta postula que la dinámica de la demanda efectiva tanto doméstica como externa en el mercado de bienes es la que determina el nivel de (des)empleo (Kaldor, 1988; Kalecki, 1995; López y Assous, 2010). Es decir, es el crecimiento de la demanda efectiva (o las ganancias realizadas) ${ }^{5}$ lo que determina la inversión. Las ganancias proveen los recursos financieros con que cuenta la empresa y a la vez señalan qué tan rentable ha sido la inversión en el pasado reciente.

En este sentido, la evolución de la demanda efectiva (es decir, de las ventas y las ganancias) determina a los inversionistas a materializar sus decisiones de inversión, mismas que impactarán el nivel de (des)empleo. Un argumento más kaleckiano, pero sin duda relacionado con la evolución de la demanda efectiva, apunta que el grado de aprovechamiento de la capacidad instalada es también un importante determinante de las decisiones de inversión (Kalecki, 1995; López y Assous, 2010). En este contexto, los movimientos de la demanda efectiva afectarán el grado de aprovechamiento de la capacidad productiva instalada, misma que también influirá en las decisiones de los empresarios a ocupar o no más trabajadores o a expandir o sustituir su maquinaria.

Así, por ejemplo, en un contexto pesimista, con la demanda efectiva ubicándose por debajo de la esperada, y con acumulación indeseada de inventarios, los empresarios se verán desincentivados a usar en su totalidad su capacidad productiva y al mismo tiempo postergarán la materialización de sus

De hecho, de acuerdo a Lavoie (2014), la visión post-keynesiana no contempla la existencia del mercado laboral.

4 En la visión post-keynesiana, el nivel de empleo determina el salario, dejando así fuera la posibilidad de que éste sea capaz de alterar la oferta y la demanda de trabajo (Badhuri, 1990; Lavoie, 2014). En este contexto, asumiendo rendimientos constantes o crecientes, el empleo y los salarios se mueven juntos.

5 Las ganancias son medidas como su tasa o como su participación en el ingreso (Badhuri y Marglin, 1990). 
decisiones de inversión, toda vez que sus ganancias habrán declinado, aumentando el desempleo como consecuencia. De mantenerse esta tendencia, es decir, sin ninguna intervención para revertirla, el nivel de desempleo aumentará.

El escenario opuesto, es decir, uno con una demanda efectiva sobrepasando la esperada, y con descenso indeseado de inventarios y ganancias en crecimiento, incentivará a los empresarios a satisfacer la creciente demanda aumentado la producción tanto a través de la puesta en marcha de su maquinaria instalada antes ociosa, y con ella empleando más mano de obra, como a través de la expansión de su capacidad productiva, implicando también mayor demanda por trabajo tan pronto como dicha inversión se materialice. Conforme este ciclo se perpetua, el nivel de desempleo decrece hasta ocupar, de ser posible, a toda la fuerza laboral.

El desempleo en la visión post-keynesiana es principalmente involuntario, debido a que sin importar el nivel de salario nominal o real, los empresarios, por un lado, de prevalecer una pobre demanda efectiva y, por lo tanto, bajas ganancias, no tendrán incentivo para demandar mayor mano de obra, con el consecuente aumento de su capacidad productiva ociosa, y los trabajadores, por el otro, pese a estar dispuestos a emplearse al salario nominal vigente no encontrarán vacantes disponibles.

En suma, la demanda efectiva determina el nivel de empleo al incidir en las decisiones de los empresarios sobre el nivel de su producción futura, misma que cubrirán o no a través tanto de mayor/menor empleo (al ocupar/desocupar la capacidad productiva existente) y/o a través de acelerar/posponer la acumulación de capital. Esta lógica puede sintetizarse en una ecuación donde la inversión inducida $\left(\mathrm{I}_{\mathrm{I}}\right)$, aquella que expande la capacidad productiva, dado el coeficiente técnico capital-producto $(\alpha)$ y el grado de capacidad productiva ocupada $(\mathrm{u})$, sea función positiva del crecimiento del producto observado $(\Delta \mathrm{Y})$, es decir de la demanda efectiva, y negativa de la tasa de depreciación del capital (d). Es decir:

$$
\mathrm{I}_{\mathrm{I}}=(\alpha+\mathrm{u}) \Delta \mathrm{Y}-\mathrm{d}
$$

De la ecuación es claro que si el producto no crece, no habrá inversión inducida, sólo se reemplazará el capital obsoleto. Pero si el producto crece, la inversión aumentará tanto para emplear la capacidad productiva desocupada como para expandir dicha capacidad.

De la propuesta post-keynesiana, la recomendación de política económica que se deriva para disminuir el desempleo radica en fomentar las decisiones de inversión de los empresarios a través de incentivar y mantener estable la 
demanda efectiva. Como es sabido, el mecanismo más efectivo a disposición de las autoridades que esta visión propone para lograr tal efecto es el uso de la política fiscal, en particular mantener el gasto público (en particular la inversión) en expansión. La inversión pública (entendida como dinero usado en la compra de bienes y servicios al sector privado destinados a proyectos de infraestructura que complementan o sustituyen a la inversión privada, como puertos, caminos, presas, escuelas, centros de salud, centros de investigación y desarrollo, etcétera) fue de hecho recomendada por Keynes por sobre el consumo público (entendida como dinero transferido a los hogares a través de salarios o bien compras de bienes y servicios a las empresas, pero como parte de su gasto corriente) para tal efecto (Smithin, 1989; Pressman, 1994; Davidson, 2007). En este sentido, la inversión pública no sólo dinamiza directamente la demanda efectiva sino además agrega a la acumulación de capital (y a otras variables como la productividad), teniendo así efectos benéficos de corto y de largo plazo.

La comprobación empírica de la perspectiva post-keynesiana hasta donde sabemos no abunda, y la existente aborda principalmente casos de economías avanzadas. Algunos de los trabajos al respecto son el de Alexiou y Pitelis (2003), Arestis et al. (2007) y Karanassou et al. (2008). Asimismo, los trabajos que estudien casos o grupos de economías en desarrollo son aún más escasos (una excepción es el estudio de Salazar y Azamar (2014) donde se toca el caso de la economía mexicana). Los resultados obtenidos en estos trabajos, tanto para economías avanzadas como para la mexicana, son uniformes en el sentido de que encuentran evidencia sobre la relación inversa entre la acumulación de capital y el desempleo.

\section{3. (DES)EMPLEO EN AMÉRICA LATINA: ¿FLEXIBILIZACIÓN LABORAL O ACUMULACIÓN DE CAPITAL?}

El inicio del siglo Xxi estuvo marcado para América Latina con el fin del nuevo boom de crisis financieras iniciado a mediados de la década de los noventa. ${ }^{6}$ A partir de entonces, es decir, a partir del inicio de la recuperación de la economía Argentina en el 2003, la región registró un periodo de estabilidad y crecimiento económico relativamente largo, interrumpido sólo hasta el 2009,

\footnotetext{
Durante la década de los noventa, América Latina tuvo importantes crisis financieras como la mexicana de 1994-95 y la brasileña de 1997-98. En 2001, Argentina también tuvo una crisis de proporciones mayores. En 2003 tuvo lugar la última crisis en la región, la de la República Dominicana.
} 
cuando el PIB se contrajo 1.6\%, consecuencia esta vez de factores exógenos, en particular la Gran Recesión global iniciada en 2007. No obstante esta caída, la recuperación fue rápida, y aunque con una tendencia a la desaceleración, el crecimiento se mantuvo en tasas positivas desde el 2010.

Paralelamente, y contrario a lo observado durante la década de los noventa, el desempleo en la región decayó lenta pero consistentemente. Así, aunque la tasa de desempleo se mantuvo alrededor de $9 \%$ a inicios de este siglo, a partir de 2004 declinó a 8.5\%, continuando una tendencia decreciente hasta llegar a una tasa cercana al 6\% en 2014 (véase gráfica 1). Es importante señalar que entre los países que observaron una tendencia decreciente significativa del desempleo destacan Argentina, cuya tasa de desempleo cayó de $18.3 \%$ en 2001 a $8.2 \%$ en 2014; Colombia, cuya tasa pasó de $16.2 \%$ en 2000 a $10.1 \%$ en 2014; Brasil, que vio declinar su tasa de desempleo de 9.5 a $6.8 \%$ entre 2000 y 2014 y Panamá, cuyo desempleo decreció de 14\% en 2001 a 4.3\% en 2014. Otros mantuvieron más bien un comportamiento estable como México, Ecuador, El Salvador, Jamaica, Honduras, Paraguay y Perú. En conjunto, insistimos, y contrario a lo que venía ocurriendo en el pasado reciente (la década de los noventa, por ejemplo), América Latina observó un decrecimiento sostenido de su tasa de desempleo a lo largo de la década de 2000.

Gráfica 1. Tasa de desempleo abierta en América Latina (\%), 1991-2014

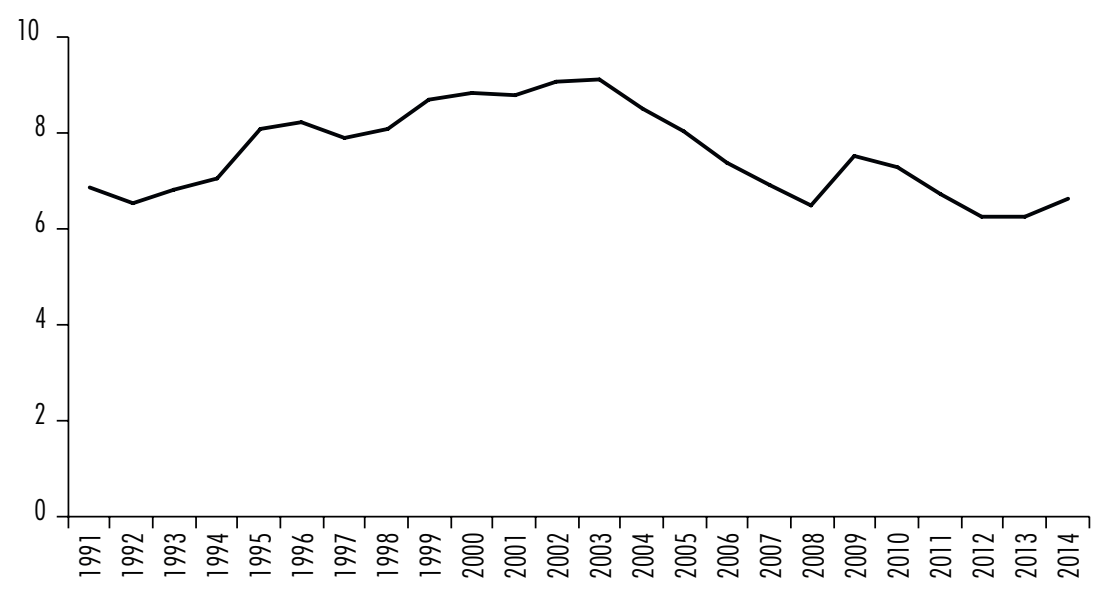

Fuente: Indicadores del Desarrollo Mundial, Banco Mundial (hittp://databank.worldbank.org/data/home.aspx) 
Ante esta evolución, la pregunta que surge es: ¿qué puede explicar este comportamiento? De acuerdo a las descripciones teóricas vertidas en la sección previa, dos son las respuestas potenciales: flexibilización del mercado laboral o crecimiento de la demanda efectiva y de la acumulación de capital. Curiosamente, la región observó ambos fenómenos, aunque ciertamente, como veremos, la flexibilización del mercado laboral fue en gran medida puesta en marcha desde la década de los noventa. El siguiente paso es ofrecer una descripción de ambos hechos.

\section{La flexibilización laboral}

La década de 2000 profundizó un proceso de reformas a las legislaciones laborales iniciada en la década de los noventa en la gran mayoría de los países de la región, cuyo objetivo fue desregular la estructura del mercado laboral. ${ }^{7}$ Muchas de estas reformas, es importante destacarlo, como en el caso de la economía mexicana, sólo vinieron a formalizar lo que en la práctica ya se estaba realizando, es decir, una flexibilidad laboral de facto (Cook, 2007). De acuerdo a Vega (2005), por otra parte, los aspectos donde más se modificó la legislación laboral con énfasis desregulador fue en los ámbitos de contrato, causas del despido, la jornada laboral, el rubro salarial y la indemnización en caso de terminación de la relación laboral. ${ }^{8}$

El cuadro 1 muestra las economías y los años en que dichas reformas fueron sucediendo. Como se observa, dicho proceso desregulador tuvo gran peso en la década de los noventa. La década siguiente, no obstante, también registró algunas modificaciones laborales, desde luego en menor número de economías. De las modificaciones ocurridas hasta 2003, es posible sintetizar que

Tokman (2008), destaca que las reformas laborales de la década de los noventa fueron parte de un segundo paquete de reformas estructurales introducidas en América Latina desde inicios de la década de los ochenta, orientadas hacia la globalización, la privatización y la liberalización (véase también Cook, 2007). En el caso particular de las reformas laborales, su objetivo era reducir los costos de despido y eliminar otras rigideces con el fin de que los bajos costos de la mano de obra atrajeran inversión extranjera directa fincando la base de su ventaja competitiva.

Antes de las reformas, los contratos prevalecientes en la región para los trabajadores del sector formal eran de tipo definitivo, mismos que incluían la obligatoriedad de avisar con anticipación el despido y otras indemnizaciones (como compensación de un mes por año trabajado hasta un techo de 11 meses). Así, el costo de despido bajo esas condiciones en América Latina era el doble que el de los países miembros de la Organización para la Cooperación y Desarrollo Económicos (OCDE) (Tokman, 2008: 69). 
Cuadro 1. Cronología de la reforma laboral en América Latina

\begin{tabular}{ll}
\hline País & Años de reformas a la legislación laboral \\
\hline Argentina & $1991,1994,1995,1996,1998,2000,2002,2003,2004$ \\
Bolivia & $1985,1994,2007$ \\
Chile & $1978,1979,1990,1994,2000,2001$ \\
Colombia & $1990,1991,1998,2000,2002$ \\
Costa Rica & 1993,2002 \\
Ecuador & $1991,1997,2000,2003,2010$ \\
El Salvador & 1994 \\
Brasil & $1991,1993,1994,1995,2000,2002,2003$ \\
Guatemala & 1995,2001 \\
Honduras & $1982,2003,2004$ \\
México & 2008,2012 \\
Nicaragua & $1995,1996,2002$ \\
Panamá & 2004 \\
Paraguay & $1992,1993,1995,2002$ \\
Perú & $1992,1993,1996,1997,1999,2002,2003,2004,2006$ \\
Uruguay & 2005 \\
Venezuela & 1990 \\
\hline
\end{tabular}

Fuente: Vega (2005) y González (2013).

“...el hecho de que en 11 de los 17 países considerados se haya dado una reforma laboral más o menos profunda con orientaciones flexibilizadoras, y que éstos representen aproximadamente el $70 \%$ del empleo asalariado de la región, parecería poner en cuestión las frecuentes afirmaciones de que la reforma laboral en América Latina no ha sido ni extensa, ni profunda..." (Vega, 2005: 13).

Como resultado de los cambios en las legislaciones laborales, América Latina incrementó relativamente rápido su nivel de flexibilidad laboral durante los años noventa. Esto se reflejó en la evolución del índice de regulación laboral de la región elaborado por el Instituto Fraser (www.fraserinstitute.org). Así, dicho índice pasó de 4.54 en 1990, a 5.31 en 1995 hasta alcanzar 5.24 en 
2000 (donde el índice se ubica en un rango de 1 a 10, siendo 10 total libertad o sin regulaciones). Durante la década de 2000, dicho índice se incrementó más lentamente, hasta llegar a 5.78 en su nivel más alto en 2009. ${ }^{9}$ Es otras palabras, el índice se movió en sintonía con los cambios hechos, pero el aumento, de acuerdo su valor, parece no reflejar la magnitud de la reforma laboral en la región. Esto incluso considerando que el índice está compuesto por variables que, como destacamos, fueron modificadas de manera importante, como las regulaciones de contratación y despido, y salario mínimo.

Debemos notar, no obstante, que de acuerdo a dicho índice, el nivel de regulación laboral de América Latina no está lejos del de países o grupos de países con un grado de desarrollo mucho más avanzado. Por ejemplo, para el conjunto de países de la Unión Europea y para 20 economías de la OCDE el índice de rigidez laboral en 2013 fue de 6.7. Este nivel es muy similar al de Alemania, de 6.5, pero muy por debajo del de Estados Unidos, de 9. Resaltan en este contexto los niveles de flexibilidad laboral de economías como Belice, Jamaica y Nicaragua, que siendo economías que se mantienen en la parte más rezagada del desarrollo económico, tiene los niveles de flexibilidad laboral más altos de la región, con 8.9, 8.4 y 7.3, respectivamente.

En suma, es claro que desde los años noventa la región en general ha sido testigo de la modificación de las legislaciones laborales, con una orientación desreguladora en concordancia con la recomendación emanada de la teoría neoclásica para disminuir el desempleo.

\section{La acumulación de capital}

Como señalamos, la reforma laboral no fue el único hecho estilizado relevante que la región vivió recientemente. En efecto, desde inicios de 2000, América Latina se caracterizó por ver un incremento sobresaliente de la acumulación de capital. En este sentido, tanto la tasa de crecimiento de la inversión como su participación en el PIB observaron un dinamismo sostenido e importante, muy por encima del observado en décadas previas. La inversión creció en promedio $4.8 \%$ entre 2000 y 2013, esto incluyendo 2009, ańo en que la inversión se colapsó $7 \%$; destaca asimismo que dicho crecimiento se mantuvo prácticamente en un espectacular $11 \%$ de 2004 a 2007. Por su parte, la in-

9

La información disponible para 1990 incluye cinco economías, para 1995 aumenta a siete, para el resto de los ańos el dato es considerando dieciocho. 
versión como porcentaje del PIB aumentó hasta cuatro puntos porcentuales, pasando de 17 a $21 \%$ del PIB entre 2002 y 2008, siendo este último un nivel sin precedente desde la década de los ochenta (véase gráfica 2). El aumento en la participación de la inversión en el PIB resulta trascedente toda vez que iniciaba a acercarse al umbral recomendado por organismos internacionales para lograr un proceso de crecimiento económico sostenido importante, esto es de entre 20 y $25 \%$ (UNCTAD, 2003).

Por otra parte, también como se observa en la gráfica 2, la evolución creciente de la inversión a partir de inicios de 2000 sólo se vio interrumpida con el inicio de la Gran Recesión global en 2007, aunque fue resentida hasta 2009. No obstante, la recuperación fue casi inmediata, retomando la senda de crecimiento en 2010. Vale la pena notar que en este contexto de acumulación de capital a la alza, la inversión pública ha jugado un papel importante a partir de 2006, cuando inicia un crecimiento que la lleva de $3.5 \%$ del piB en ese ańo a $4.7 \%$ en 2013. Finalmente, debemos recordar que el dinamismo de la inversión es fundamental en la evolución del рIв, del tal suerte que su estabilidad y crecimiento ayudó a que el producto creciera en promedio $3.2 \%$ en el periodo 2000-2013. Este es un crecimiento que, sin ser espectacular, no es nada desdeñable para una región marcada, en décadas pasadas, por la inestabilidad y el lento crecimiento.

Grática 2. Formación bruta de capital en América Latina, 1990-2014

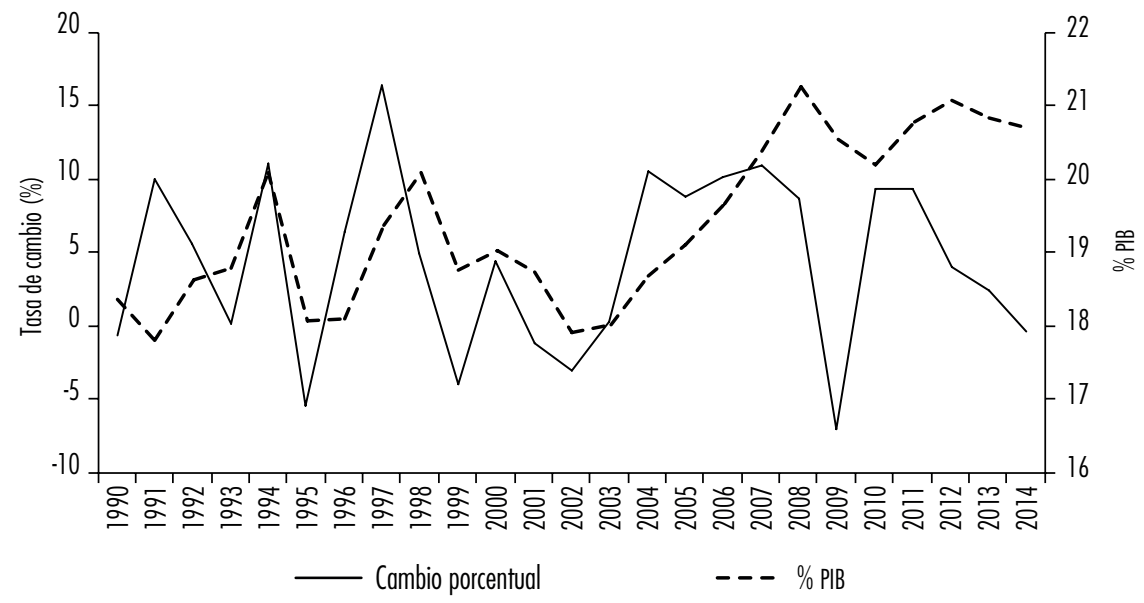

Fuente: Indicadores del Desarrollo Mundial, Banco Mundial (hitrp://databank.worldbank.org/data/home.aspx) 
Aunque está fuera de los alcances de este trabajo, es importante señalar que el principal, aunque desde luego no único, responsable del buen momento económico que la región vivió se debió al repunte económico mundial, impulsado por la dinámica de economías en desarrollo, como China. Lo anterior, para una región que predominantemente mantiene una orientación exportadora de bienes primarios (alrededor de 50\% del total), significó un aumento importante en la demanda por sus bienes, siendo sin duda un factor de relevancia en el crecimiento económico observado. Este momentum fue aprovechado hasta que la economía global se colapsó a partir de 2007, sin que hasta la fecha pueda recuperar los niveles de crecimiento previos.

El aumento sobresaliente de la formación bruta de capital en la región sería, de acuerdo a la visión post-keynesiana, el elemento principal que sustenta el decremento sostenido del desempleo.

\section{El ejercicio econométrico}

Ante la disyuntiva que plantean los hechos estilizados, procedemos a realizar un ejercicio econométrico con datos de una muestra de 18 economías latinoamericanas para el periodo 2000-2013 mismo que, insistimos, observó un sostenido decrecimiento de la tasa de desempleo. Las economías que integran la muestra son: Argentina, Belice, Bolivia, Brasil, Colombia, Costa Rica, Ecuador, El Salvador, Guatemala, Honduras, Jamaica, México, Nicaragua, Panamá, Paraguay, Perú, República Dominicana y Venezuela, mismas que fueron seleccionadas principalmente en función de la disponibilidad de datos, con el objetivo de identificar empíricamente qué fue lo que causó el abatimiento del desempleo en América Latina durante el periodo objeto de estudio. La idea, en este sentido, es estimar mediante técnicas de panel la siguiente especificación:

$$
u_{i t}=\alpha_{i}+\beta_{k} x_{k i t}+\varepsilon_{i t}
$$

donde $u_{i t}$ es la tasa de desempleo abierta urbana del país $i$ en el periodo $t$, 2000-2013, $\alpha_{i}$ representa el efecto fijo que captura los determinantes no observados del desempleo de cada país y $x_{i t}$ es el vector que contiene las variables explicativas, donde por un lado está el índice de regulación laboral (b) y por, el otro, los componentes de la demanda agregada; es decir, el consumo privado $(c p)$, las exportaciones $(x)$, el gasto de gobierno $(g g)$ y la formación bruta de 
capital $(k)$. Las variables de la demanda están expresadas como porcentaje del PIB y fueron transformadas en logaritmos, lo mismo que la tasa de desempleo. Las variables asociadas a la regulación laboral se mantuvieron en niveles. Esperamos que las variables explicativas tengan signo negativo, indicando una relación inversa entre cada variable y la tasa de desempleo. Los datos del índice regulación laboral fueron obtenidos de la base de datos del índice de Libertad Económica del Instituto Fraser (www.fraserinstitute.org), mientras que los componentes de la demanda agregada fueron obtenidos de los Indicadores de Desarrollo Mundial del Banco Mundial (www.databank.worldbank.org).

Es importante destacar que decidimos realizar la estimación a través de la técnica de efectos fijos para, como es usual en este tipo de ejercicios, incluir la heterogeneidad de las economías de la muestra. Asimismo, como la base conforma un panel desbalanceado, usamos los errores estándar robustos para corregir por posible heterocedasticidad que pueda afectar los resultados de la regresión (Asteriou y Hall, 2011).

El cuadro 2, columna I, reporta los resultados de la estimación básica que explica el desempleo en función del índice de regulación laboral compuesto y de la acumulación de capital. La idea es intentar capturar el efecto de ambas variables en la evolución del desempleo toda vez que, como señalamos, la reforma laboral ya estaba en marcha en dicho periodo, aunque en algunos casos aún sin haber modificaciones laborales ya existía de facto, y al mismo tiempo se observó un crecimiento de la acumulación de capital importante.

Como podemos ver, ambas variables tienen el signo esperado, no obstante, sólo la acumulación de capital es estadísticamente significativa. Este resultado, se mantiene a lo largo de las estimaciones posteriores (columnas II y III). Es importante notar, de hecho, que la respuesta del desempleo a la acumulación del capital es grande, indicando que por cada porciento que como porcentaje del PIB aumenta la acumulación de capital, la tasa de desempleo decrece en $1 \%$.

En la columna II, se muestran los resultados al incorporar los componentes restantes de la demanda agregada, esto es el consumo público $(g g)$ y privado $(c p)$ y las exportaciones $(x)$. La intención aquí es echar luz sobre si algún otro de estos componentes ha sido crucial en explicar el desempleo. Como puede verse, ninguna de estas variables es capaz de explicar el desempleo al ser estadísticamente no significativas, no obstante el consumo privado y las exportaciones tienen el signo esperado. De manera interesante, el gasto público tiene signo positivo, algo inesperado en el contexto post-keynesiano. El índice de regulación laboral compuesto mantiene el signo negativo y sigue siendo estadísticamente no significativo. 
Ahora bien, como señalamos, los aspectos donde más se modificó la legislación laboral en América Latina fue en los ámbitos de contrato, causas del despido, la jornada laboral, el rubro salarial y la indemnización en caso de terminación de la relación laboral. Para cerciorarnos si a través de alguno de estos rubros la flexibilidad laboral afectó el desempleo, decidimos dejar de lado el índice de flexibilidad laboral compuesto e introducir en su lugar los componentes que lo integran. Dichos componentes son cinco y miden lo siguiente: $b i$, las regulaciones de contratación y salario mínimo; bii, las regulaciones por contratación y despido; biii, la negociación centralizada de contratos colectivos; biv, las regulaciones por hora y costo de contratación por trabajador; finalmente $b v$, que mide el costo obligatorio por despido.

La columna III muestra los resultados de esta estimación. Como puede verse, la acumulación de capital mantiene el signo, tamaño y significancia estadística. Tampoco cambian los resultados en relación al resto de los componentes de la demanda agregada. Respecto a las medidas individuales de regulación laboral, bii y $b v$ tienen el signo esperado, pero sólo la primera es estadísticamente significativa. Es decir, las medidas enfocadas a flexibilizar las contrataciones y el salario mínimo han tenido un efecto positivo en la tasa de desempleo. El resto de las medidas tiene signo positivo y son estadísticamente no significativas.

Finalmente, ante la relevancia de la acumulación la capital para explicar el desempleo, decidimos incorporar a la estimación el ingreso per cápita real para probar si, como destaca el argumento post-keynesiano, la inversión responde principalmente a la evolución de la demanda efectiva. En otras palabras, si la inversión varía en función de la demanda efectiva, entonces el (des)empleo lo hará también. La columna IV incluye como variables explicativas al logaritmo del ingreso per cápita en términos reales $(y p c)$ y al índice de regulación laboral compuesto. De manera notable, como puede apreciarse, la tasa de desempleo responde prácticamente en la misma medida en que lo hace con la acumulación de capital. Es decir, por cada incremento porcentual del ingreso, la tasa de desempleo disminuye en la misma medida. Por su parte, la regulación laboral compuesta sigue siendo irrelevante para el desempleo.

En suma, nuestros resultados indican que la acumulación de capital ha sido la responsable principal de la caída del desempleo en América Latina durante el periodo 2000-2013. En este sentido, la visión teórica post-keynesiana es respaldada por la evidencia empírica. La flexibilización laboral también ha incidido a través de las modificaciones a las regulaciones de contratación y de salario mínimo, aunque su impacto parece minimizarse ante un periodo de crecimiento económico importante. 
Desempleo en América Latina: ¿̇flexibilidad laboral o acumulación de capital?

Cuadro 2. Determinantes de la tasa de desempleo (U), 2000-2013

\begin{tabular}{|c|c|c|c|c|}
\hline $\begin{array}{l}\text { Variables } \\
\text { independientes }\end{array}$ & I & $\|$ & III & IV \\
\hline \multirow[t]{2}{*}{ k } & $-1.049^{\star \star}$ & $-1.113^{\star \star}$ & $-0.947^{\star \star}$ & \\
\hline & $(0.161)$ & (0.168) & $(0.172)$ & \\
\hline \multirow[t]{2}{*}{$b$} & -0.009 & -0.019 & & -0.022 \\
\hline & $(0.013)$ & $(0.165)$ & & $(0.044)$ \\
\hline \multirow[t]{2}{*}{$g g$} & & 0.180 & 0.233 & \\
\hline & & $(0.370)$ & (0.324) & \\
\hline \multirow[t]{2}{*}{$c p$} & & -0.616 & -0.757 & \\
\hline & & $(0.605)$ & $(0.597)$ & \\
\hline \multirow[t]{2}{*}{$x$} & & -0.169 & -0.017 & \\
\hline & & $(0.237)$ & $(0.283)$ & \\
\hline \multirow[t]{2}{*}{ bi } & & & 0.015 & \\
\hline & & & $(0.032)$ & \\
\hline \multirow[t]{2}{*}{ bii } & & & $-0.086^{\star \star}$ & \\
\hline & & & $(0.031)$ & \\
\hline \multirow[t]{2}{*}{ biii } & & & 0.046 & \\
\hline & & & $(0.042)$ & \\
\hline \multirow[t]{2}{*}{ biv } & & & -0.023 & \\
\hline & & & $(0.042)$ & \\
\hline \multirow[t]{2}{*}{ bv } & & & 0.0002 & \\
\hline & & & $(0.010)$ & \\
\hline \multirow[t]{2}{*}{ урс } & & & & $-1.272^{\star \star}$ \\
\hline & & & & $(0.267)$ \\
\hline \multirow[t]{2}{*}{ Constante } & $5.36^{\star \star}$ & $8.20^{\star \star}$ & $8.29 * \star$ & $13.5^{\star \star *}$ \\
\hline & $(0.473)$ & (3.82) & (3.53) & (2.24) \\
\hline Observaciones & 238 & 238 & 183 & 245 \\
\hline Wald (joint) & 0.000 & 0.000 & 0.000 & 0.000 \\
\hline Wald (dummy) & 0.000 & 0.000 & 0.000 & 0.000 \\
\hline AR(1) (p-value) & 0.002 & 0.003 & 0.003 & 0.004 \\
\hline
\end{tabular}

Notas: Los errores robustos estándar se muestran en paréntesis; ${ }^{\star} y^{* \star}$ denotan significancia estadística al 1 y 5 por ciento, respectivamente.

Fuente: elaboración propia. 
Estos hallazgos empíricos tienen importantes implicaciones de política. En primera instancia, nos permiten sugerir que es de poca trascendencia para los empresarios al momento de decidir cuánta más mano de obra ocupar el nivel desregulación laboral ya que el mismo empequeñece o desaparece ante la relevancia de la demanda efectiva. De hecho, si el objetivo de la flexibilidad laboral es reducir el costo de la mano de obra, entonces enfatizar en dicha estrategia puede resultar contraproducente para estimular el empleo toda vez que menores salarios reducirán la demanda efectiva y, por lo tanto, la acumulación de capital. No olvidemos que los salarios son tanto un costo de producción como un componente fundamental de la demanda. La evolución de los salarios en la demanda en este sentido es crucial en economías en vías de desarrollo, como las latinoamericanas, pues aún no han transitado a la dependencia permanentemente de la demanda externa, vía la producción y exportación de bienes con elevada elasticidad ingreso de la demanda. Pero incluso en economías donde el sector externo juega un papel relevante en la demanda y el crecimiento económico, los salarios son también muy importantes, al empujar la demanda interna.

En segundo lugar, si se desea acercar a las economías de Latinoamérica al pleno empleo, son necesarias políticas que incentiven el crecimiento de la demanda, lo cual repercutirá en la acumulación de capital de manera positiva. Una de las pocas alternativas inmediatas en manos de los hacedores de política, especialmente en un contexto de contracción de la demanda externa, es el uso de la inversión pública, como lo ha venido haciendo la región desde 2006. Como señalamos, la inversión pública no sólo tiene efectos inmediatos en los niveles de demanda, también los tiene en el mediano y largo plazos, al expandir la capacidad productiva, e impactar positivamente en los niveles de productividad (Pressman, 1994; Galstyan y Lane, 2009). De hecho, una estrategia expansiva de la inversión pública, insertada en una estrategia industrial bien definida, permitiría dar el salto hacia la producción de bienes sofisticados. Este proceso generaría sin duda mayor empleo.

\section{CONCLUSIONES}

Durante el periodo 2000-2013, América Latina fue testigo de un decrecimiento sostenido de la tasa de desempleo acompańado de dos fenómenos que pocas veces ocurren de manera simultánea: una reforma laboral con ánimo flexibilizador fuertemente hecha en los años noventa y reforzada en el 2000, y el aumento significativo de la acumulación de capital. Cada uno de estos 
fenómenos podría ser capaz, por sí sólo, de dar cuenta de la caída del desempleo, a través de dos escuelas teóricas opuestas en ese sentido. La neoclási$\mathrm{ca}$, que propone a la flexibilización laboral como mecanismo para resolver problemas de desempleo y la visión post-keyenesiana, que sustenta que es el aumento de la acumulación de capital, como resultado del crecimiento de la demanda efectiva, como la vía para disminuir el desempleo.

Partiendo de dicho marco, en este trabajo, usando datos de 18 economías de la región y técnicas de panel, se investigó cuál variable, y por lo tanto cuál visión teórica, es más factible de dar sustento a lo observado. Los resultados sugieren que es la demanda y la acumulación de capital quienes explican principalmente el desempeño del desempleo durante el periodo de estudio. Es decir, es la visión post-keynesiana quien ofrece el marco teórico adecuado para explicar la caída del desempleo. Las medidas enfocadas a flexibilizar las contrataciones y el salario mínimo han tenido también un efecto positivo en la tasa de desempleo, pero al parecer dicho efecto se minimiza en un contexto de crecimiento económico como el estudiado.

De los resultados, es posible sugerir que para reducir el desempleo, es necesario sostener el crecimiento de la acumulación de capital, siendo la vía principal la dinámica de la demanda efectiva. Para este fin, la visión post-keynesiana recomienda, en un contexto de contracción de la demanda externa y de lento crecimiento de la productividad y, por lo tanto, de los salarios, aumentar la inversión pública. De manera relevante, esto es algo que la región ha venido haciendo desde 2006. En este sentido, la idea sería mantener e incluso aumentar el crecimiento de la inversión pública pero vinculándola en una estrategia de política industrial con el fin de transitar hacia la producción de bienes con alto valor agregado, mismos que tienen elevada demanda en el mercado externo. En un contexto así, es factible que el desempleo mantenga su tendencia decreciente.

\section{BIBLIOGRAFÍA}

Aleksynska, M. (2014), "Deregulating Labour Markets: How Robust is the Analysis of Recent IMF Working Papers?", Conditions of Work and Employment Series núm. 47, International Labour Office, Geneva.

Alexiou, C. y C. Pitelis (2003), "On Capital Shortages and European Unemployment: A Panel Data Investigation”, Journal of Post Keynesian Economics, vol. 25, núm. 4, pp. 613-640. 
Arestis, P., M. Baddeley y M. Sawyer (2007), "The Relationship between Capital Stock, Employment and Wages in Nine Emu Countries", Bulletin of Economic Research, vol. 59, núm. 2, pp. 125-148.

Asteriou, D. y S. Hall (2011), Applied Econometrics (2nd ed.), Reino Unido, Palgrave-MacMillan.

Ayala, A., J. Cuñado y L. Gil-Alana (2012), "Histéresis del desempleo: evidencia empírica para América Latina", Revista de Economía Aplicada, vol. 15, núm. 2, pp. 213-233.

Bernal-Verdugo, L., D. Furceri y D. Guillaume (2013), "Banking Crises, Labour Reforms, and Unemployment", Journal of Comparative Economics, vol. 41, núm. 4, pp. 1202-1219.

, D. Furceri y D. Guillaume (2012), "Labour Market Flexibility and Unemployment: New Empirical Evidence of Static and Dynamic Effects", IMF Working Paper, 12/64.

Betcherman, G. (2014), "Labor Market Regulations. What do We know about Their Impacts in Developing Countries?", Policy Research Working Paper no. 6819, The World Bank.

Bhaduri, A. (1990), Macroeconomia: la dinámica de la producción de mercancías, México, Fondo de Cultura Económica.

Bhaduri, A. y S. Marglin (1990), "Unemployment and The Real Wage: The Economic Basis for Contesting Political Ideologies", Cambridge Journal of Economics, vol. 4, núm 4, pp. 375-93.

Blanchard, O. (1997), Macroeocnomía, Reino Unido, Prenctice-Hall.

Carlin, W. y D. Soskice (2006), Macroeconomics: Imperfections, Institutions and Policies, Reino Unido, Oxford University Press.

Cheng-Hsun, L., K. Nai-Fong y Y. Cheng-Da (2008), "Nonlinear vs. Nonstationary of Hysteresis in Unemployment: Evidence from OECD economies", Applied Economics Letters, núm. 15, pp. 905-909.

Cook, M. (2007), The Politics of Labor Reform in Latin America. Between Flexibility and Rights, Estados Unidos, The Pennsylvania State University Press.

Cross, R., H. Hutchinson y S. Yeoward (1990), "The Natural Rate, Hysteresis, and The Duration Composition of Unemployment in the U. S.", Quarterly Journal of Business and Economics, vol. 29, núm. 2, pp. 89-116.

Crivelli, E., D. Furceri y J. Toujas-Bernaté (2012), "Can Policies Affect Employment Intensity of Growth? A Cross-Country Analysis", IMF Working Paper núm. 12/218.

Hicks, J. (1937), "Mr. Keynes and the 'Classics'. A suggested Interpretation”, Econometrica, vol. 5, núm. 2, pp. 147-159. 
Davidson, P. (2007), John Maynard Keynes, Reino Unido, Palgrave Macmillan. Dias, N. (1995), "Paradox of Hysteresis and Real-wage Flexibility in Australia”, Journal of Post-Keynesian Economics, vol.17, núm. 4, pp. 503-514.

Dritsaki, C. y M. Dritsaki (2013), "Hysteresis in Unemployment: An Empirical Research for Three member States of European Union", Theoretical and Applied Economics, vol. 20, núm. 4, pp. 35-46.

Furceri, D. (2012), "Unemployment and Labour Market issues in Algeria", IMF Working Paper no. 12/99.

Galí, J. y T. Monacelli (2016), "Understanding the Gains of Wage Flexibility: The Exchange Rate Connection", American Economic Review, vol. 106, núm. 12, pp. 3829-3868.

Galstyan, V. y P. Lane (2009), "The Composition of Government spending and The Real Exchange Rate", Journal of Money, Credit and Banking, vol. 41, núm. 6, pp. 1233-1249.

González, J. (2013), Reforma laboral: algunos apuntes para el análisis legislativo, Documento de Trabajo no. 148, Centro de Estudios Sociales y de Opinión Pública de la Cámara de Diputados (México)..

Kalecki, M. (1995), Teoría de la dinámica económica, México, FCE.

Kaldor, N. (1988), "The Role of Effective Demand in the Short and Long Run”, en A. Barrère (ed.), The Fundations of Keynesian Analysis, London, Macmillan Press.

Karanassou, M., H. Sala y P. Salvador (2008), "Capital Accumulation and Unemployment: New Insights on the Nordic Experience", Cambridge Journal of Economics, vol. 32, pp. 977-1001.

Keynes, J. (2000), La teoría general de la ocupación, el interés y el dinero, México, FCE.

Lavoie, M. (2014), Post-keynesian Economics. New Fundations, Reino Unido, Cheltenham Edward Elgard.

Layard, R., R. Jackman y S. Nickell (1991), Unemployment: Macroeconomic Performance and the Labour Market, Oxford, Oxford University Press.

León-Ledesma, M. y P. McAdam (2004), "Unemployment, Hysteresis and Transition”, Scottish Journal of Politycal Economy, vol. 51, núm. 2, pp. 377 401.

López, J. y M. Assous (2010), Michal Kalecki, New York, Palgrave-Macmillan. Maurer, M. y D. Nivia (1994), "La histéresis en el desempleo colombiano", Cuadernos de Economía, vol. 14, núm. 21, pp. 223 - 239.

Mednik, M., C. Rodríguez y I. Ruprah (2012), "Hysteresis in Unemployment: Evidence from Latin America", Journal of International Development, vol. 24, núm. 4, pp. 448-466. 
Nickell, S., L. Nunziata y W. Ochel (2005), "Unemployment on the OECD since the 1960s. What do We Know?", Economic Journal, vol. 115, pp. $1-27$.

Phelps, E. (1972), Inflation Policy and Unemployment Theory: The Cost-Benefit Approach to Monetary Planning, primera edición, The MacMillan Press, London.

Pressman, S. (1994), "The Composition of Government spending: Does It Make any Difference”, Review of Political Economy, vol. 6, núm. 2, pp. 221-239.

Salazar, C. y A. Azamar (2014), "Flexibilidad y precarización del mercado de trabajo en México", Política y Cultura, núm. 42, pp. 185-207.

Samuelson, P. y R. Solow (1960), "Analytical Aspects of Anti-inflation Policy”, American Economic Review, vol. 50, núm. 2, pp. 174-194.

Siebert, H. (1997), "Labour Market Rigidities: at the Root of Unemployment in Europe", Journal of Economic Perspectives, vol. 11, núm. 3, pp. 37-54.

Smithin, J. (1989), "The Composition of Government Expenditures and the Effectiviness of Fiscal Policy", en J. Pheby (ed.), New Directions in Postkeyensian Economics, Inglaterra, Edward Elgar, pp. 209-227.

Tokman, V. (2008), "From the Concensus Reforms to Reforms for the Protected and Inclusive Employment", IDS Bulletin, vol. 39, núm. 2, pp. 69-78. unctad (2003), Trade Development Report. Capital Accumulation, Growth and Structural Change, United Nations, New York and Geneva.

Vega Ruíz, M. (2005), La reforma laboral en América Latina: 15 años después. Un análisis comparado, Organización Internacional del Trabajo, Lima. 